\title{
Horizons/Théâtre
}

Revue d'études théâtrales

\section{Dramaturgies et manifestations spectaculaires. Problématiques du théâtre en Afrique}

\section{Sélom Komlan Gbanou}

\section{(2) OpenEdition}

1 Journals

Édition électronique

URL : https://journals.openedition.org/ht/917

DOI : $10.4000 /$ ht.917

ISSN : 2678-5420

Éditeur

Presses universitaires de Bordeaux

\section{Édition imprimée}

Date de publication : 31 décembre 2018

Pagination : 10-34

ISBN : 979-10-300-0318-5

ISSN : 2261-4591

\section{Référence électronique}

Sélom Komlan Gbanou, «Dramaturgies et manifestations spectaculaires. Problématiques du théâtre en Afrique ", Horizons/Théâtre [En ligne], 13 | 2018, mis en ligne le 01 janvier 2019, consulté le 17 mai 2022. URL : http://journals.openedition.org/ht/917 ; DOI : https://doi.org/10.4000/ht.917

\section{cc) (†) $\odot$}

La revue Horizons/Théâtre est mise à disposition selon les termes de la Licence Creative Commons Attribution - Pas d'Utilisation Commerciale - Pas de Modification 4.0 International. 


\section{SÉLOM KOMLAN GBANOU}

Sélom Komlan Gbanou a enseigné aux universités de Bremen et de Bayreuth en Allemagne, à l'École Nationale de Théâtre du Canada. Actuellement Professeur titulaire à I'Université de Calgary, il est le fondateur et le directeur de publication de la revue de langue française Palabres. $\|$ est l'auteur de plusieurs ouvrages et articles sur les littératures et les théâtres d'Afrique et de la Caraibe. Parmi ses publications : Ahmadou Kourouma, "Allah n'est pas obligé ", Paris, Honoré Champion, 2013 ; Un théâtre au confluent des genres, Frankfurt am Main, London, 2002.

Mail : sgbanou@ucalgary.ca

Résumé: Les travaux sur le théâtre en Afrique ne s'émancipent que rarement des travaux de François Victor Equilbecq qui, au début du $x x^{e}$ siècle, se targuait, dans un élan eurocentrique, du privilège d'avoir apporté le théâtre aux " indigènes d'Afrique ». Dès lors l'idée que le théâtre africain est $d$ 'invention européenne perdure, faisant perdre de vue la dimension de l'essence humaine de jeu qui est à l'origine de toute pratique dramaturgique. L'objet de la présente étude est de réfléchir sur cette part de partition

Abstract : The work on Africa theatre seldom emancipates itself from the works of François Victor Equilbecq who at the beginning of the XXth century boasted, in a Eurocentric impulse, of the privilege of having brought theatre to the "natives of Africa". Since then, the idea that African theatre is of European invention continues, thereby obscuring the dimension of the human essence of play which is at the origin of all dramaturgical practice. The objective of this study is to reflect on this engagement, the re-presentation of the visible de jeu, de re-présentation du visible et de I'invisible, de Soi et de l'Autre, du concret et de l'abstrait qui caractérise le théâtre en Afrique. II interroge les pratiques culturelles et cultuelles dans leur rapport au corps social et au corps de l'individu dans une perspective de spectacle, le rôle et la place du théâtre écrit dans les langues occidentales et les nouvelles formes d'adaptation du théâtre africain au cinéma.

Mots-cLés : dramaturgisation, identité collective, enseignement, téléthéatre.

and the invisible, of Self and the Other, of the concrete and the abstract that characterizes theatre in Africa. It questions cultural and cult practices in their relationship to the social body and the body of the individual in a dramatic perspective, the role and place of theatre written in Western languages and the new forms of adaptation of African theatre to cinema.

KEYwORDS: dramaturgization, collective identity, teaching, teletheatre. 


\section{Dramaturgies et manifestations spectaculaires. Problématiques du théâtre en Afrique}

C'est en Afrique, au Maghreb et plus spécifiquement dans le village de Chebika en Tunisie que le célèbre anthropologue Jean Duvignaud a pu découvrir des réalités socioculturelles d'un ordre nouveau qui vont définitivement bouleverser ses idées sur le théâtre européen hérité de l’Antiquité grecque, et qu'il croyait, comme nombre de chercheurs européens, universel. Dans ce village en effet, Duvignaud se rend à l'évidence qu'en théâtralisant leur existence, les habitants, qui vivent pourtant dans un grand dénuement matériel, arrivent à se réinventer et à éprouver le bonheur à l'échelle individuelle et sociale, à travers la force et la magie de la représentation. Duvignaud en arrive alors à des hypothèses fort riches qui serviront de matières premières à son essai : Les Ombres collectives (1965), qui reste à ce jour l'un des regards les plus pertinents sur la pratique théâtrale en tant qu'expression et manifestation de l'individu et de la société. Pour Duvignaud :

Le théâtre est donc bien plus que le théâtre. Sans doute, un des plus anciens de tous et, quand on nous demande d'énumérer les figures les plus illustres de l'humanité, ce sont des noms de dramaturges qui nous viennent d'abord à l'esprit Eschyle, Shakespeare, Molière. Mais c'est un art enraciné, le plus engagé de tous les arts dans la trame vivante de l'expérience collective. (p. 11)

Et si le théâtre est plus que ce que l'on voit et le lieu d'où s'exerce ce regard, c'est dire qu'il y a nécessité de s'interroger, non pas sur l'existence ou non du théâtre en Afrique, mais plutôt sur la dramaturgie de l'existence telle qu'elle fonctionne au sein même des peuples africains auxquels la critique occidentale croit toujours avoir « apporté » le théâtre. Esthétique et éthique sociale, la pratique du théâtre - mieux la pratique spectaculaire - relève d'un mécanisme collectif de recherche de la permanence du cercle social. Ainsi, tout comme au sein des autres peuples et civilisations du monde, la pensée sociale, culturelle en Afrique ne se développe que dans la conscience profonde et l'esprit de l'être humain dans une harmonie constamment recher- 
chée entre le sacré et le profane, la tradition et le modernisme, l'oralité et l'écriture. Le théâtre est, avant toute autre considération, un jeu social, une forme de contemplation spirituelle et exotérique du monde qui fait entrevoir, dans les contradictions de la vie, une réévaluation consentie des conflits entre l'idéal et le réel, le Mal et le Bien, le visible et l'invisible, le ludique du pédagogique. Chaque peuple est alors présent dans son théâtre de même que chaque théâtre est le miroir du peuple qui le produit. Par-delà la littérature dramatique qui relève, en ce qui concerne surtout l'Afrique, d'un autre registre, le théâtre comme pratique sociale est au fond un rituel ontologique où l'individu, la communauté se projettent dans une espèce de voyance dans laquelle le passé, le présent, le devenir sont pensés sur une scène pour une (re) découverte du Moi individuel aux prises avec le Nous collectif. Il cristallise la vie dans ses angoisses, ignorances, croyances et convictions à travers des effets représentatifs bien travaillés, fondés sur une grande valeur poétique.

Que ce soit dans les pratiques rituelles comme la possession auxquelles une certaine critique voudrait enlever toute charge de théâtralité, ou des manifestations festives saisonnières ou encore dans les danses au clair de lune, ces spectacles privilégient des dispositions scéniques comme le travail de distribution des rôles, d'aménagement des espaces scéniques et scénographiques, le choix des accoutrements, le maquillage, les masques, etc. qui mettent en relief un sens partagé du jeu et des enjeux sociaux comme un moment intense de raffermissement des liens communautaires. Ces dispositions qui combinent savoir et savoir-faire ont la particularité, comme c'est le cas dans toutes les formes de théâtres que compte le monde : le Vaudeville, Le Nogaku, le Kabuki, le Wayang kulit, etc., de divertir. Cependant, et c'est un point important au centre de cette étude : la pesanteur idéologique de la colonisation (française ou anglaise) a différemment affecté, mais en profondeur, la pratique théâtrale en Afrique déjà vouée au diktat et à l'hégémonisme du regard d'Aristote.

\section{Et revoilà Aristote... !}

« Aristote a déthéâtralisé, désenchanté le théâtre. Libérer la scène contemporaine, c'est redécouvrir les théâtres ritualisés, ludiques, musicaux¹. »

L'erreur perdure tant qu'elle est confortable. Et en cela, la pratique théâtrale en Afrique a fait les frais de la vision ethnocentrique du théâtre, trop longtemps entretenue, qui a consisté à tout ramener à Aristote et à ainsi méconnaître ou à nier la préhistoire et l'histoire de l'art de la scène sur le continent. 
Ce parti pris a réduit les différentes pratiques spectaculaires en lesquelles les autres cultures du monde fondent ce qui constitue leur théâtre en un avatar, en une pâle copie du legs de la Grèce antique. Et pourtant, « l'aspiration à la détente » et à la beauté 2 qui constitue, selon Johan Huizinga, le moteur de tout jeu social ne saurait se réduire à une quelconque spécificité culturelle, au risque de perdre de vue ce qui en constitue le mécanisme et le fonctionnement. Aristote, avec sa focalisation sur la tragédie, n'a pas su informer sur la pratique théâtrale, dans son intégralité, de son époque en tant qu'art scénique, d'autant plus que les dionysies, les thrènes, les orgies au Dieu bachique qui s'inscrivent parfaitement dans cette aspiration à la détente et à la réinvention esthétique des scènes de vie quotidienne pourraient rapprocher le théâtre de la Grèce antique d'autres formes similaires de jeux sociaux aux relents de théâtre. Du coup, en voulant servir Aristote à toutes les cultures, on en est arrivé à niveler le sens et les fonctions de tout ce qui, avec le temps, a convergé vers le théâtre dans sa conception occidentale actuelle. Pour Huzinga,

la communauté archaïque joue comme jouent l'enfant et l'animal. Cette opération, dès l'origine, est riche des éléments propres au jeu : ordre, tension, mouvement, solennité, enthousiasme. Dans une phase plus évoluée seulement de la vie sociale s'associe à ce jeu la conception que quelque chose s'y trouve exprimé: une image de la vie. Ce qui était un jeu dépourvu d'expression verbale prend une forme poétique ${ }^{3}$.

Cette « phase plus évoluée » est-elle l'apanage d'un peuple, d'une culture ? Tout le malentendu à propos des pratiques théâtrales en Afrique est là dans cette méconnaissance (voulue ?) des paramètres esthétiques du passage de la forme archaïque de la magie, des rituels, des danses sacrées, etc. au processus d'imagination créatrice qui transforme le jeu originel en une structure sociale codifiée. C'est dire qu'en Afrique, et cela bien avant l'impérialisme linguistique du français, de l'anglais ou du portugais, l'espace social est toujours en soi un espace scénique où l'individu, acteur dans l'âme, mime le réel, l'imaginaire, la transcendance, en cherchant à tout instant à reproduire ses gestes, à engager son corps par la musique, la danse, les processions dans le plaisir constitutif des ambiances festives, saisonnières, religieuses, profanes de la société. Par l'intention consciente du jeu, du plaisir collectif, le rituel peut se faire théâtre, c'est-à-dire ce lieu de partages et de complicité entre regardants et regardés comme dans les rites dionysiaques grecs.

Si Jean Duvignaud énonce les expériences de l'Europe dans les représentations de sa trame de vie sociale, évoque l'idée « d'invention d'un théâtre », ce n'est pas pour parler de l'émergence spontanée, sous une quelconque tu- 
telle culturelle étrangère, mais pour insister sur les processus esthétiques qui interviennent dans le travail collectif de théâtralisation de l'existence.

C'est un fait surprenant, estime Duvignaud, que l'extraordinaire effervescence dramatique qui, deux fois dans notre histoire, se développe - ou, plus exactement, explose - en Grèce antique de la fin $d u I^{e}$ siècle à la fin du v viècle avant J.-C., en Europe, entre 1580 et 1640. Cela est si surprenant que l'on peut raisonnablement parler de l'invention d'un théâtre, pour ces deux périodes...

La démarche que nous avons adoptée nous demande d'admettre une présentation systématique, et non évolutive. Cela veut dire que, du point de vue de la typologie sociologique, les formes d'expériences esthétiques de l'Antiquité et des $\mathrm{XVI}^{e}-\mathrm{XVII} I^{e}$ siècles hispano-anglais doivent être présentées conjointement, dans la mesure où il s'agit d'un genre de création dramatique qui survient au moment où les structures des sociétés traditionnelles (patriarcales en Grèce, féodales en Europe) se trouvent radicalement modifiées et que la dramaturgie apparaît comme inséparable de la conscience collective et individuelle de ces changements ${ }^{4}$.

Or, du point de vue historique et sociologique, les structures traditionnelles ont connu aussi d'importants bouleversements à travers des guerres, des conquêtes, les catastrophes, de grandioses manifestations, etc. où les personnes humaines, les divinités sont exaltées à travers des théâtralités qui évoluent dans le temps par un potentiel esthétique constamment enrichi de la matière sociale. En ce qui concerne l'Afrique, comme on peut le lire dans travaux de Robert Cornevin, de Jacques Scherer, d'Alain Ricard et bien d'autres critiques, l'idée « d'invention du théâtre » semble plutôt suggérer la naissance spontanée du théâtre en Afrique par la générosité d'une Europe soucieuse de partager son sens de jeu social avec le reste du monde - les colonies - dépourvu de cette dimension. Pourtant, Jean Duvignaud semble avoir perçu le danger d'une telle aristotélisation de la pratique théâtrale, qui consiste à en faire un art universel porté par l'écriture ; d'où cette approche :

Quelle que soit l'importance que l'on accorde à une tradition théâtrale (qui n'existe que pour l'historien), on peut constater que la pratique théâtrale avec ses genres dérivés change selon que la mise en scène de la personne humaine proposée et les émotions qu'elle entraine, se modifient, selon que l'expérience esthétique se situe dans le monde féodal, la société monarchique où les sociétés contemporaines. Le rôle et la fonction du théâtre eux-mêmes paraissent profondément transformés par ces changements, au point que l'on devrait se demander parfois si l'on a affaire au même art ${ }^{5}$. 
De son côté, Bakary Traoré, dans son essai Le Théâtre négro-africain et ses fonctions (1958), n’a pas manqué de souligner l'impact négatif de cette subordination du théâtre africain - toujours réduit à la phase archaïque de l'instinct ludique - aux normes définitoires prônées à une échelle universelle par l'Occident. Il dispose :

L'on a peu à peu - la dégradation de la tradition aidant - réduit le théâtre de l'Afrique noire à « quelques tableaux » d'une danse gratuite autour d'un feu de bois, au son du tam-tam. Et peu à peu, l'Africain a aussi acquis cette conviction ${ }^{6}$.

Il y a lieu de souligner que même dans la structure ludico-spirituelle de ces «quelques tableaux», s'effacent les frontières entre sacré et profane, mythe et réalité, collectif et individuel, éthique et esthétique par un travail de reconstitution du temps et de l'Espace. En naissant du sacré, comme le théâtre grec, romain et plus tard français ou anglais, le théâtre en Afrique s'inscrit dans une dynamique culturelle, une conscience de la permanence et de l'immanence dans les relations entre d'une part les différents membres de la communauté et d'autre part entre la communauté dans son ensemble et les diverses représentations de ses divinités et de ses ancêtres. Dans cette perspective, les rites initiatiques, de guérison, les pratiques vaudoues, les soirées de conte, impliquent une fonction et une fiction à la fois de cette permanence et de cette immanence par des jeux, des pratiques spectaculaires sous formes de structures plus ou moins organisées où l'engagement divin qu'implique à l'origine la dimension sacrée devient une implication d'Acteurs dans une mise en scène de la parole. Le rite finit toujours par se faire un mécanisme de formalisation de la transcendance, de l'Invisible, un processus par lequel l'on cherche un support humain à l'Irréel car les manifestions anthropomorphiques du divin ont ce pouvoir secret de rassurer les humains tout en renforçant leur crainte du mystère. Même le conte n'échappe pas au processus de déritualisation, comme le montre Bernard Agudze-Vioka, dans son travail sur le conte dans le golfe de Guinée, Mensonges en or (2001), où conteur et public isolent l'univers et le temps du récit du périmètre du sacré en l'exotérisant pour mieux s'y projeter. Le conteur est ici un acteur, un comédien dans le sens moderne du terme dont les modalités de la parole s'inscrivent dans une trame dialogique avec le public relégué au rang de spectateur-acteur ${ }^{7}$ :

Le conteur : «Écoutez le conte! » Le public : « Conte! Arrive! 》

Conteur: « le conte prend son vol, vole, vole, plane, plane, plane, longtemps et se pose sur le Roi, sur l'Araignée, sur le Lion, sur la Panthère et sur la Fille des bois. » 
Le public: «Nous étions làne fait jamais défaut dans le conte. »

Conteur : « Calebasse-rongée-par-les termites ne prend pas de vin ; Mettez-y du vin, le vin aussitôt se fermente. »

Le public: « Deux personnes intelligentes ne font pas de partage ; il faut qu'il y en est un, moins intelligent. »

Le public : « Le rhumatisme dit que s'il en vient aux prises avec le tibia, s'il ne l'aplatit pas, il le gondole, s'il ne le tord pas, il le couvre de mille et une fistules. »

Tam-tam! Tam-tam! Tam-tam! Tam-tam!

Conteur : «Nous étions donc là comme nous sommes là ce soir autour de ce $\mathrm{feu} \ldots \gg^{8}$

Il y a ici une harmonie dans le système des codes qui désintègre en jeu et en fonctions dramatiques le conte traditionnel que dit le Grand Prêtre appelé Babalawo, le Bokonon ou encore le Houbonon dans une configuration ésotérique limitée au couvent sans d'autres publics que l'initié ou celui qui vient consulter les Dieux. Le Grand Prêtre ritualiste n'est pas un acteur. Sa parole professe un savoir ésotérique qu'il récite sans intention de jeu d'où l'absence de toute fonction phatique qui sollicite l'attention de son destinataire. C'est dans le travail de déritualisation, d'exotérisation du sacré, de l'ésotérique, de redistribution des signes, incarnés par le conteur, que se trouve l'esprit de la dramaturgie dans les pratiques spectaculaires dans la plupart des cultures en Afrique où cohabitent imperceptiblement sacré et profane. Le signe, connecteur culturel, rend présent ce qui est absent. Ainsi, une nouvelle disposition de la sémiotique théâtrale permettrait, sans nul doute, de déterminer son implication, sa participation à un ensemble structuré de pratiques ludiques, ses sens connotatifs, ses liens avec les autres signes de l'environnement social pour une relecture de l'anthropologie sociale du continent. En somme, à force de chercher à conformer l'univers théâtral africain à celui, déjà bien simplifié par l'écriture, de l'Europe, on a fini par en faire des pratiques spectaculaires des formes toujours primitives du théâtre occidental, sans grande valeur esthétique. Mais ce « nouveau théâtre » de langue française - pour ce qui concerne l'Afrique dite francophone - tant vanté, est-il pour autant une instance en laquelle se retrouve volontiers le spectateur africain ? 


\section{Un théâtre ou des théâtres ? Les écueils du théâtre à la française}

Nos contemporains ont oublié que le théâtre est un jeu, dont ce qui se joue sur la scène n'est qu'un élément, comme les cartes qu'on retourne sur le tapis au poker; mais que le partenaire conserve par-devers soi une autre série de cartes, des cartes secrètes par rapport auxquelles les cartes retournées prennent seulement leur vraie valeur'.

C'était en ces termes que Pierre-Aimé Touchard, administrateur général de la Comédie-Française, plaidait pour plus d'exigence dans le rapport du public, du critique au théâtre. Il reprochait ainsi l'esprit de complaisance avec lequel le mouvement intérieur et la force évocatrice et incantatoire du jeu théâtral sont perçus par le spectateur qui n'en comprend pas souvent les règles. Touchard compare le spectateur de théâtre à celui d'un match de football qui suivrait, distrait et sans grand intérêt, les mouvements de la balle sans se soucier des principes qui régissent les déplacements des joueurs, attendant le but - ultime moment - pour exploser de joie ou de déception. Ces critiques sont formulées il y a plus d'un demi-siècle, à l'encontre du public européen formé et initié, pour ainsi dire, aux règles classiques du jeu théâtral hérité de l'Antiquité grecque et pratiqué avec habileté et aisance depuis des siècles autant par des acteurs que par des spectateurs qui ont en commun un sens concerté de l'activité théâtrale. Transposées dans le champ de la dramaturgie africaine, ces remarques prennent plus d'ampleur car le public y est mis devant un jeu dont il ne possède pas toujours les outils d'appréciation. Le théâtre à l'occidentale est un art nouveau qui a profondément modifié l'attitude spectatrice du public africain en y introduisant de nouvelles modalités qui enjoignent d'appréhender le spectacle hors du traditionnel fond commun et anonyme de la religiosité et du festif dans lequel tout le monde est plus ou moins acteur. Envisager une telle perspective suppose que l'on prenne autrement en compte, dans l'activité dramaturgique, de la réception, des relations entre le public et le spectacle. La négligence de cet aspect important est une lacune dans les approches sur la pratique théâtrale en Afrique car ne permettant pas de mieux cerner le phénomène dramatique dans sa fonctionnalité. Ayayi Togoata Apédo-Amah sera le premier à consacrer une réflexion à la question, non du statut du spectateur africain, mais de ses modalités de théâtre dans le théâtre, il interroge le travail du public dans l'effectivité de la scène. En effet, dans son article « L'attitude spectatrice du public africain ${ }^{10} \gg$, A. T. Apédo-Amah part de l'hypothèse que le théâtre dans les sociétés africaines ne pourrait être véritablement apprécié que sur le plan de l'affectivité dans laquelle la perception individuelle est constamment confrontée à la 
perception collective. Le théâtre est un moment festif où, mis au contact de la vie, le public s'y sent engagé comme acteur dans l'événement, en se donnant en spectacle « à soi-même et aux autres ». Dans ce schéma festif, le créateur de théâtre (acteur ritualiste, comédien-conteur) est un ordonnateur de fêtes dont la prestation s'inscrit dans une dynamique socialisante qui mobilise autour d'un pathos collectif ou plutôt qui renforce le système de régulation de la communication et de la communion sociales autour des problèmes, de la joie, des questions, des rapports à la transcendance, etc. L'idée sous-jacente de l'analyse d'Apédo-Amah qui couvre l'espace du Golfe de Guinée (Togo, Bénin, Ghana, Côte d'Ivoire) est qu'il y a une pratique du spectateur qui, en soi, est un théâtre et, en même temps, une instance de modélisation du spectaculaire et de ses énonciations. Il s'agit d'une présence massive au jeu intimement liée au facteur culturel :

L'expérience esthétique de chaque peuple est inséparable de sa culture. À preuve, au sein du même public, on note parfois une nette différence d'attitude entre l'élite intellectuelle et le public populaire. Cette différence tient essentiellement à la formation à l'Occidentale de ces intellectuels qui, par conséquent, du fait du phénomène de l'acculturation, se trouvent déphasés par rapport à la culture populaire locale ${ }^{11}$.

La notion d'acculturation est à prendre au sens d'autoreprésentation au miroir de l'Autre, un processus plus ou moins inconscient de pérennisation de l'entreprise coloniale française qui vise à créer chez le colonisé une conscience de néant culturel à travers un système éducatif fondé sur l'idée de culture dominante à élever au rang d'universel.

Du coup, en indexant le spectacle à une nouvelle tradition, le public africain l'indexe à des réalités nouvelles d'auteur, de metteur en scène, de salle, de genre, de comédiens, etc. acquises sur le tard et qui le soumettent à des exigences inattendues dont les conséquences, plus ou moins insidieuses, ont profondément modifié la conception du spectacle, de son enseignement et même de l'attitude du spectateur.

Le théâtre « moderne » est d'invention récente en Afrique et son fonctionnement tient de son enseignement dans les établissements secondaires et à l'université - parce qu'il s'agit de la littérature dramatique, du théâtre sans sa version papier - où tout est réduit à la tyrannie de la littérature dramatique, au support linguistique et non aux valeurs fondamentales du spectacle pourvu d'exigences esthétiques implicitement partagées par le public. Ainsi, né à l'école, le théâtre africain écrit, du moins dans le sens où on l'entend généralement aujourd' hui, est un théâtre approximatif qui dans une large mesure se 
fait, implicitement, le prolongement du théâtre à l'européenne dont les règles du jeu ne sont pas encore suffisamment ancrées dans les habitudes du grand public.

Très souvent limitées au registre du thématique dans le théâtre écrit, la plupart des approches s'attachent à rechercher une adéquation de la scène africaine aux canons de la comédie, du drame, du mélodrame ou de la tragédie, catégories dramatiques qui n'affectent pas, dans leurs structures classiques, le public africain confronté à des problèmes sociopolitiques et économiques immédiats. Cette approche conceptuelle par laquelle le théâtre est présenté en Afrique comme un art nouveau d'invention récente, malgré son dynamisme, postule en arrière-plan une bipolarité dans l'art de la scène. D'un côté, le théâtre littéraire acquis aux schèmes des discours critiques constamment renouvelés, de l'autre un théâtre de masse extrêmement varié, soumis aux expériences de sa société avec laquelle elle partage le médium de la langue et les complicités énonciatives. Aussi les notions de théâtre francophone, de théâtre anglophone ou lusophone sont extrêmement réductrices de la dynamique théâtrale dans son ensemble en Afrique, car il semble de plus en plus évident que les théâtres populaires avec les nouvelles technologies de communication : le DVD, et l'Internet, les réseaux sociaux (Facebook, WhatsApp surtout) ont pris une sérieuse avance sur le théâtre à l'occidentale.

\section{Dramaturgies et enseignement}

La pratique et l'enseignement du théâtre ne jouissent pas du même statut dans les mondes anglophone et francophone en Afrique. Ainsi, on éprouve quelques difficultés à esquisser un tableau comparatif de la pratique théâtrale dans les deux aires linguistiques qui tiennent compte des similitudes et des singularités esthétiques et thématiques en raison, d'une part, des barrières linguistiques, de l'autre, de la filiation culturelle que les pays africains entretiennent entre eux et avec leurs anciens colonisateurs, même si la démarche n'en garantit pas une approche plus globale.

Ce qui pose problème, ce n'est guère la pédagogie mais son objet. Certes, dans la diversité de ses formes, les études théâtrales ont toujours suscité des querelles ${ }^{12}$ entre partisans de la scène et du texte, mais ce qui en rend l'approche difficile dans l'univers africain c'est la coexistence de ce qui répond aux normes définitoires du théâtre à l'européenne avec des pratiques spectaculaires en lesquelles se retrouve entièrement et avec délectation le public. Ainsi, la mention « théâtres africains » au pluriel renvoie, de manière 
explicite, à cette pluralité problématique de l'activité théâtrale fondée sur deux réalités.

La première consiste à tenir compte de la dualité entre le théâtre à texte et le théâtre-canevas. Le théâtre à texte, dans la plupart du temps, est un théâtre sélectif qui limite son public aux locuteurs de la langue d'écriture. Doublement intéressant du point de vue d'une pratique pédagogique et d'une dynamique sociale, il est souvent un théâtre de ville et dans lequel le dramaturge est en même temps metteur en scène, acteur et pour le critique propose, avec bonheur, de prospecter le texte au détriment du spectacle et de sa réception. Il nécessite des séances de répétition alors que le théâtre-canevas, théâtre de l'improvisation par excellence, puise sa matière et sa dynamique dans la rencontre de la compétence d'un ou de plusieurs acteurs avec la compétence d'un public. Il opère par une restructuration, une adaptation de certains lieux communs que sont le conte, les légendes et reprend les schèmes de la morale sociale dans un ton qui varie de la satire à la tristesse mais avec ceci qu' il joue sur la connivence et la complicité des codes implicitement partagés.

La deuxième réalité est l'opposition évidente entre les deux premières formes de pratique théâtrale à un troisième pôle, relativement jeune mais dynamique qui combine la scène, la télévision et le cinéma dans un jeu virtuel aux effets saisissants. C'est le téléthéâtre qui est avant tout un théâtre de studio se réalisant dans un nouvel esprit de mise en scène dans lequel interviennent ingénieurs de son et d'images, opérateurs sur ordinateur, cameramen, éclairagistes, maquilleurs, storyboardeurs, etc., et qui bouleverse profondément les rapports entre la scène et le public mais aussi le statut de la scène et du spectateur.

On ne pourrait argumenter que sur la base de quelques hypothèses qui relèveraient moins de l'ordre de l'esthétique que du socio-politique, car les rapports que le théâtre entretient avec son public sous-tendent plus particulièrement ceux de la langue, de la sociologie des peuples, de la conception esthétique et philosophique du genre, éléments sur lesquels l'enseignement devrait pouvoir trouver des méthodologies d'interprétation adéquates. Or, l'introduction du théâtre dans les programmes d'enseignement dans les lycées et universités en Afrique ne semble pas tenir suffisamment compte du fait que le théâtre est l'interface entre le corps social et sa propre mise en scène et celle du monde. L'enseignement du théâtre s'est fait un prolongement de l'idéologie coloniale de «l'invention du théâtre en Afrique ». La démarche s'inscrit dans la doctrine coloniale de l'assimilation dont Henri Brunschwig retrace le processus dans Mythes et réalités de l'impérialisme colonial français ${ }^{13}$ à travers le 
souci de grandeur et d'expansion de la civilisation sur le primitivisme, d'être les Grecs du monde comme le disait Victor Hugo dans Choses vues, suite à la conquête de l'Algérie. Une telle conception de l'enseignement comme doctrine d'assimilation trouvait déjà, en ce qui concerne l'art de la scène, sa mise en pratique chez François Victor Equilbecq, administrateur des colonies, qui, après avoir nié l'existence du théâtre africain, pouvait se targuer du privilège de l'avoir inventé :

Je ne me fais pas un titre de gloire d'avoir tenté le premier une cuvre théâtrale exclusivement indigène. [...] je suis heureux néanmoins d'avoir ouvert la voie et d'avoir, moi étranger à leur race comme à leur terre, inauguré un théâtre national pour nos indigènes de l'Ouest-Africain-Français ${ }^{14}$.

Si les propos de Equilbecq traduisent un ethnocentrisme excessif dont l'objectif est de nier l'existence d'une dynamique théâtrale autour de laquelle s'organiserait la vie des communautés africaines, il est à noter qu' ils ont inspiré des tendances de rejet de ce qui, chez les peuples colonisés, tenait lieu de pratiques théâtrales. Par la suite, les missions culturelles conduites en Afrique francophone pour y évaluer la pratique théâtrale partiront du principe que le théâtre, dans sa conception européenne, n'existe pas en Afrique et qu'il faille l'inventer. En fait l'ouvrage de l'ethnologue français Equilbecq a été rédigé en 1914 mais sa publication sera retardée par la Première Guerre mondiale et la mort de l'auteur en 1917. Robert Cornevin, qui entre en possession du manuscrit en 1966 à travers la personne de Madame de Maistre, le publie en $1974^{15}$. L'hypothèse de l'invention du théâtre en Afrique par le truchement de la colonisation développée par Equilbecq, reprise d'ailleurs par Robert Cornevin en 1970 dans son ouvrage Le Théâtre en Afrique et Madagascar ${ }^{16}$, est donc à analyser à la lumière de l'événement enclencheur de la dramaturgisation de l'Autre en face du colonisateur : l'être africain. Cet événement enclencheur dont l'École normale fédérale William Merleau-Ponty est le berceau pose des modalités d'émergence d'une perception dramaturgique de l'Africain et de son environnement.

Créée en 1903 à Saint-Louis au Sénégal, puis transférée en 1913 à l'île de Gorée toujours au Sénégal, l'École normale fédérale William Merleau-Ponty avait pour mission de former des polytechniciens au service de l'administration coloniale. Le rôle déterminant qu'elle va, par la suite, jouer dans l'avènement d'une dramaturgie africaine de langue française tient de deux facteurs : d'abord de l'engouement personnel du gouverneur général lui-même pour les arts du spectacle et ensuite du désir de scénarisation de l'Autre, déjà inscrit dans les fondements de la colonisation. 
Avant son affectation en 1935 à l'École normale fédérale William MerleauPonty, Charles Béart avait dirigé l'École primaire supérieure de Bingerville en Côte d'Ivoire où il fut fortement marqué par le talent d'improvisation et de jeu de ses « écoliers indigènes » dont Amon d'Aby, Aka Bilé, Bernard Dadié. Voici comment Charles Béart reconstruit l'événement :

Un mardi de novembre, commente-t-il, le tailleur était dans la cour pour essayer les uniformes et les élèves étaient très mécontents, car le tailleur n'ayant pas trouvé les boutons de corozo traditionnels, les avait remplacés par des boutons de cuivre qui faisaient ressembler les élèves à des gardes-cercle. Tout à coup un élève, Aka Bilé, passe la ceinture de son pantalon par-dessus la veste neuve, fait un sabre d'un bâton et, dans le sabir que parlent les indigènes non évolués de la Basse-Côte, interpelle ses camarades [...] Ses camarades lui donnèrent la réplique, la mauvaise humeur s'était envolée. J'avais entendu de mon bureau et je dis à Aka Bilé : ceci est d'excellent théâtre, il faut nous jouer quelque chose comme cela un samedi (Cornevin, $1984: 19$ ).

Le théâtre de Bingerville venait ainsi de naître. Charles Béart quitte donc la Côte d'Ivoire avec un bagage qui servira de tremplin au théâtre pontin qui connut sa consécration en 1937, année où l'école est invitée à l'Exposition internationale de Paris avec à l'affiche les 12 et 17 août les pièces Sokamé (jouée par les élèves dahoméens) et Les Prétendants rivaux (par les élèves de la Côte d'Ivoire).

Les passages ci-dessus cités introduisent dans l'approche de la dramaturgie africaine des éléments importants qui participent de la sociologie du théâtre au sens où la conçoivent Johan Huizinga et Roger Caillois. Il s'agit essentiellement de l'altérité de soi dans une pratique d'interprétation du monde à travers l'instinct de jeu. Cet instinct de jeu inhérent à la nature humaine Huizigan parle de l'homo ludens ${ }^{17}$ - postule qu'il y a des éléments du monde réel qui animent ce désir de transformation et de transposition de ce que l'on voudrait revoir, revivre dans le cours irréversible des choses. Ainsi s'explique la notion de « représentation » dont l'usage fort ambigu ne rend pas suffisamment compte du caractère événementiel du contexte dramatique ${ }^{18}$ individuellement et collectivement vécu. Les motifs générateurs de «l'excellent théâtre » dont parle Béart s'inscrivent en effet dans ce que Caillois nomme le ludus et le mimicry ${ }^{19}$. Le ludus qui désigne la tendance à organiser et structurer dans un esprit de jeu le vécu et le rêvé est un penchant de la pensée humaine créatrice à réinventer le réel par la représentation en lui enjoignant une atmosphère de délectation et de divertissement pour un regard extérieur. Il implique, de ce fait, une mise en scène de soi dans le besoin d'être 
autre, un Soi imaginé dans la procédure d'une rupture avec le réel. Tel est le deuxième principe de la pensée humaine créatrice, auquel Caillois donne le nom de mimicry. S'inscrivant dans la perspective de Johan Huizinga en ce qui concerne l'origine ludique du théâtre, Roger Caillois estime que l'activité théâtrale s'épanouit dans cette conjonction de la conscience de jeu et du désir de transformation pour atteindre une simulation esthétique de la réalité. Dans les propos de Charles Béart, il apparaît que c'est de l'instinct de jeu des élèves pour surmonter une situation désapprouvée que naquit ce mouvement improvisé et fantaisiste qui rompt d'avec la discipline et les règles de docilité.

Mais pour qui les élèves de l'École Primaire Supérieure de Bingerville, en ce mardi de novembre, se mettaient-ils en scène ? Sans entrer dans le détail des mécanismes de «représentation » de la classe dominée, de ce que Augusto Boal appelle le «théâtre de l'opprimé », il est nécessaire, dès l'abord, de remarquer que le jeu théâtral fut, pour les élèves de l'École primaire supérieure de Bingerville, une mise en scène d'eux-mêmes dans une optique d'autodérision et de catharsis. L'objectif de leur jeu improvisé n'était nullement une rébellion contre le système colonial dont ils subissaient les exactions mais simplement de rendre banale et supportable une situation contre laquelle, dans la réalité, ils ne pouvaient rien.

Au niveau du jeu, ce qui s'exprime dans l'acte performatif n'est pas différent de ce que la culture des élèves exprime, car comme l'observe Caillois, l'instinct de jeu est intrinsèquement lié à la culture et son effectivité passe par des « rapports étroits de compensation et de connivence avec celle- $\mathrm{ci}^{20} »$. Il s'était agi pour les jeunes «indigènes » de puiser dans leurs ressources culturelles un acte créateur qui, même s'il n'est pas un art constitué dûment régi par des règles, est chargé de sens et porteur de cette force de transcendance que l'instinct ludique véhicule. Ainsi, par le ludus, les élèves s'inventent une exigence d'ordre intellectuel qui substitue à la situation réelle une image reconstruite qui procure jouissance et satisfaction aussi bien à ceux qui s'y livrent qu'à ceux qui regardent dont Béart. La réaction de ce dernier : « ceci est d'excellent théâtre, il faut nous jouer quelque chose comme cela un same$\mathrm{di} \gg$ traduit, en d'autres termes, l'essence du jeu telle que le concevait Johan Huizinga, c'est-à-dire comme « une action libre, sentie comme fictive et située en dehors de la vie courante, capable néanmoins d'absorber totalement le joueur ; une action dénuée de tout intérêt matériel et de toute utilité ; qui s'accomplit en un temps et dans un espace expressément circonscrits ${ }^{21} \gg$ avec pour motif l'accomplissement d'un acte destiné à être vu. Le jeu improvisé, ainsi que son organisation en théâtre par la suite, est un mode de transfert de 
la réalité à l'ordre du fictionnel où le donné à voir conduit à trois fonctions dramaturgiques :

a) la conscience du jeu comme attribut de l'humain capable d'exprimer, de manière opératoire, le potentiel culturel d'une communauté donnée. On retrouve ici le concept de sémiosis ininterrompue ${ }^{22}$ développé par Peirce pour désigner ce désir de connaissance et de transformations qui porte l'humanité dans son ensemble ;

b) la représentation comme transformation dans des limites de temps et d'espace avec des déterminations esthétiques de l'objet rendu signe par la scène ${ }^{23}$;

c) le théâtre du représentant, son propre dévoilement comme signe à même de dynamiser des savoirs. En se représentant, les élèves de Bingerville et plus tard de l'École normale fédérale William Merleau-Ponty se présentent et présentent à travers eux leur milieu.

Il est tout aussi remarquable, que même si le jeu de la représentance n'est pas formalisé comme un art soumis à des règles étanches, il opère par une combinaison de signes qui en fait une dramaturgisation du réel, et dont la singularité est de permettre de « mieux comprendre la vie ${ }^{24} \gg$. Le plaisir théâtral procuré à l'administration coloniale par la représentation et l'autoreprésentation des indigènes s'est transmué en un exercice de perception de l'Être noir dans sa totalité en substituant à l'objet représenté, le microcosme du représentant. Le ludus fait alors figure de cet « instant prégnant » dont parle Lessing dans son Laocoon (1766) et qui est ce moment spécifique où toute l'histoire et l'historicité du personnage se trouvent condensées en une image. Le regard exotique porté sur cette image ainsi conçue comme porteuse à la fois du passé, du présent et de l'avenir de l'Africain conduit Charles Béart et l'administration coloniale de l'Afrique occidentale française (AOF), dont le Sénégal était la capitale, à un mimétisme absolu qui ne pouvait autoriser à voir derrière le jeu scénique des élèves qu'une simple scène de l'Autre qui ne laisse pas de place à un regard sur le dispositif esthétique en œuvre dans ces créations.

En fait le théâtre pontin est devenu un théâtre de l'indécidé et de l'indécidable dans lequel, pris au piège de leur propre altérité, les acteurs n'ont pas leur propre autorité sur leur théâtre devenu l'ego autonome de l'énonciation. Ils sont, en quelque sorte, le référent de leur jeu, l'objet du regard de l'Autre, sujet et objet de leur propre mise en scène, s'inscrivant, ipso facto, dans l'idéologie coloniale par son penchant à une représentation caricaturale des coutumes africaines. Koffi Kwahulé, dans son essai Pour une critique du théâtre ivoirien contemporain ${ }^{25}$, justifie le théâtre pontin comme un passe-temps 
favori, un « délassement digestif » pour les administrateurs coloniaux et l'élite locale constituée des chefs traditionnels possédant quelques rudiments de la langue française et quelques anciens élèves de l'École d'administration William-Ponty. En effet, les jeunes auteurs, futurs polytechniciens, fascinés par la culture dominante, incorporent la rhétorique de leur infériorité et exhibent, devant les exigences de supériorité du maittre, une altération de leur propre altérité. Dépouillés d'eux-mêmes dans une dialectique de la représentance - le représentant et l'objet représenté -, les jeunes « indigènes » se mettent en demeure de vilipender leurs propres cultures car il est presque impossible de représenter l'image que l'on se fait de sa propre altérité sans les apories de la restriction et de la chosification. Des pièces comme Kondé Yao de Kondé Yao et Une couronne aux enchères de Main Aoussi fustigent la chefferie traditionnelle dans ses structures originelles jugées rétrogrades. D’autres à l'instar de Entraves (Amon d'Aby), Mon mari (Koffi Gadeau), Situation difficile et Serment d'amour de Bernard Dadié partent de l'intrusion de l'économie monétaire et de marché dans la société pour faire un procès acerbe de la société traditionnelle. La colonisation comme thème d'écriture était sinon absente du moins superficielle dans ce théâtre pontin dont les textes importaient moins que leur potentiel de dramaturgisation d'un monde loin du monde. Tel pourrait être le sens de la participation des élèves de l'École fédérale William-Ponty à l'Exposition internationale de Paris en 1937.

On relève ici le statut de l'exhibition en tant que mise en scène consciente ou non de soi formulée par Thimotee Mitchell dans l'essai Colonizing Egypt à propos de l'Exposition internationale de Paris en 1889, où ce que le regard occidental mettait en valeur, ce fut moins l'objet représenté (exposé) que le représentant. Le théâtre du représentant implique, de ce fait, qu'il y a psychologisation de l'objet représenté et sa substitution par une altérité imaginée du représentant, considération qui n'a rien à voir avec le topos du Theatrum Mundi utilisé de l'antiquité grecque à Balzac comme paradigme absolu de la condition humaine ${ }^{26}$. De là naît la dramaturgisation du colonisé dont la pratique culturelle est convertie en une «sémantique sociale » fortement réductrice dans la construction des identités collectives. Guy Michaud, reprenant certaines hypothèses de Jacques Berque ${ }^{27}$, initiateur du concept de l'identité collective, explique que l'identification collective se fait le « lieu même de la production de la sémantique sociale ${ }^{28} \gg$ où, mis en scène et en signe, le sujet ainsi placé dans le registre de l'ethnotype devient à la fois sujet et objet, ici regardant et regardé, selon qu'il se perçoit ou est perçu. Car la dramaturgisation en tant que regard de spectateur porté sur l'Autre en scène est déterminée par le mode de perception qui porte la signification du représen- 
té. Le système d'acquisition de savoir sur le JE social auquel étaient soumis les élèves de William-Ponty les confronte à des repères identificatoires qui les soumettent à leur tour à un drame psychologique : celui de se voir sous le regard d'un Autre. Ils devaient, pendant les vacances, recueillir dans leurs différents milieux les schèmes d'une identification de masse de leur culture. Le théâtre pontin est alors devenu le lieu d'élaboration d'un imaginaire qui légitime implicitement les préjugés auxquels est soumis l'acteur amené à mettre en place un système d'interprétation d'un ethnotype : une mentalité collective au sens où Jacques Berque formule le concept.

Les différentes problématiques constitutives du théâtre du représentant se comprennent, de ce fait, comme un paradoxe de la représentation dans laquelle le regard instituant de l'Autre vient conditionner sa signification comme signe :

L'identité, avance Edmond Marc-Lipiansky, se situe dans ce jeu d'images qui captent l'individu, dans ces clichés où il peut ne pas se reconnaître mais où les autres le figent, dans ces représentations qu'il veut croire extérieures, mais qui l'interpellent du dedans de lui-même $e^{29}$.

Il apparaît que le théâtre pontin dans les conditions de son émergence (le ludus) s'inscrivait plus dans une dynamique idéologique de perception du dedans et du dehors du sujet du jeu que dans un travail esthétique de valorisation du langage théâtral. Ce que les élèves croient être une fiction devient fatalement la mise à jour d'un regard porté sur un ayant-été atypique dont on cherche ou récuse le sens dans la confrontation à la différence. L'hypothèse formulée par Éric Landowsky sur la quête de l'identité comme conséquence d'une crise d'altérité trouve ici tout son sens. Landowsky considère que la construction de l'identité comme expérience d'une existence sémiotique nécessite la présence d'un IL, des Autres, d'Eux :

Ce qui donne forme à ma propre identité, ce n'est pas seulement, en effet, la manière dont, réflexivement, je me définis (ou tente de me définir) par rapport à l'image qu'autrui me renvoie de moi-même, c'est aussi la manière dont, transitivement, j'objectivise l'altérité de l'autre ${ }^{30}$.

Tout semble indiquer que l'effectivité de la représentation passe par une double scène : la scène de soi comme sujet et la scène de l'autre comme figure du distant. Cette double scène participe, tant au niveau des élèves que de l'administration coloniale, de l'élaboration d'un statut de la différence. Pour Charles Béart et les autres, il s'agit, en convertissant le jeu improvisé en une dramaturgie constituée, de situer les colonisés par rapport à une norme 
de référence qui se construit ses propres représentations du mode de vie et de pensée de l'Autre. Dans cette perspective, il faut le relais d'une instance sémiotique au sens de sujet qui se charge d'effectuer les opérations de référencement et de sélection des comportements de cet Autre en face pour un achèvement du statut de la différence. La mobilisation des élèves pour représenter les us et coutumes de leurs origines dans une démarche plutôt anthropologique problématise la question de la construction de l’altérité du colonisé. Désormais, c'est par son propre regard que le colonisé se perçoit. Désormais, c'est lui qui « fixe l'inventaire des traits différentiels qui, de préférence à d'autres possibles, serviront à construire, à diversifier et à stabiliser le système des "figures de l'Autre" qui sera, temporairement ou durablement, en vigueur dans l'espace socioculturel considéré31 ${ }^{1}$ »

L'image singulière de l'étrangeté, la non-reconnaissance de Soi, les tensions et les déchirements intérieurs vécus entre l'Altérité de Soi et l'Altérité du Maître (le colonisateur) deviennent cause, en ce qui concerne l'Afrique francophone, d'un autre drame dont les anciens pontins et la dramaturgie africaine de langue française, dans son intégralité, contrairement au monde anglophone, prendront brutalement conscience bien plus tard.

\section{Le paradigme anglophone}

Ce qui pourrait donner plus d'éclairage sur le théâtre et sa pratique en Afrique viendrait des approches qui proposeraient des voies d'entrée directe à la scène théâtrale car les études sur la littérature dramatique comme procédés de compréhension du théâtre en Afrique ne semblent pas cerner véritablement la scène dans ses différentes fonctionnalités. Il y a lieu d'élargir les angles d'approches du théâtre, d'en faire ce que Jean-Pierre Miquel nomme une « consommation culturelle ${ }^{32}$ » qui dépasse le périmètre restreint de la langue pour s'étendre à un corps social plus vaste. Certes, les travaux sur le théâtre en Afrique ne le prouvent pas assez, d'autant plus qu'il s'agit d'un aspect dont on ne prend conscience que tardivement, en raison d'un programme d'enseignement aliénant qui fait du théâtre un art importé en Afrique, mais il existe un dialogue fécond entre le théâtre anglophone et le théâtre francophone dans un mouvement qui alterne l'influence, l'intertextualité et la transculturalité. En d'autres termes, le théâtre francophone se nourrit énormément des expériences de son homologue anglophone et pour cause.

En Afrique anglophone, le théâtre se présente prioritairement comme un enjeu national et un art à consommation locale par opposition au statut de théâtre d'exportation auquel fait souvent penser le théâtre francophone. En 
effet, que ce soit au Nigeria, au Kenya, en Tanzanie, en Gambie, au Ghana, la pratique théâtrale n'est pas a priori tenue par des exigences d'un update occasionnel ou permanent qu'impose l'horizon d'attente des lieux périphériques de légitimation et de reconnaissance qu'est la France avec des Prix (Prix RFI, prix Beaumarchais...), des festivals annuels (Francophonies en Limousin, Festival d'Avignon, Festival de Blaye...).

Même sur le plan d'une dramaturgie purement écrite, l'Afrique anglophone fait figure de pionnière à travers une tradition de valorisation des langues locales favorisée par le système de décolonisation du Indirect Rule initié par le gouverneur du Nord-Nigeria Sir Frederick Lugard. Cet aspect dont les missionnaires allemands en poste au Ghana dans ce qui s'appelait l'Eweland entre 1834 et 1919 avaient déjà été les apôtres par un intense travail de codification de ces langues ${ }^{33} \mathrm{~s}^{\prime}$ inscrivait logiquement dans la perspective d'une littérature religieuse. Cependant, la création en septembre 1926, à Londres, de l'Institut international des langues et civilisations africaines, va tracer une ligne de partage entre le littéraire et le religieux. Pour ce faire, un travail préliminaire devra déterminer dans les régions africaines les idiomes de ralliement entre les différents dialectes et l'élaboration d'une orthographe pratique. Le but de ce travail préliminaire est d'aboutir au lancement d'un concours littéraire dont la première édition date de 1930. Naturellement, les pays anglophones et les anciennes colonies allemandes comme le Cameroun, le Togo britannique, la Namibie, plus préparés à écrire les langues «indigènes », prennent un avantage certain sur les colonies françaises ${ }^{34}$. C'est dans ce cadre qu'en 1933 l'ewephone du Ghana Ferdinand Kwasi Fiawoo écrit la tragédie Toko Atolia (La Cinquième Lagune) en langue ewe qui remporte le premier Prix. Cette œuvre qui connaîtra quatre éditions fut traduite en allemand en 1936 par Diedrich Westermann, membre du jury du concours, avec la collaboration de Reinhold sous le titre de Die Fünfte Lagune; en 1943 paraît la version anglaise The Fith Landing Stage réalisée par l'auteur même. La qualité de la pièce, son inscription aux canons du genre de la tragédie ${ }^{35}$ en ont fait un classique bien avant l'Exposition internationale de Paris de 1937 considérée la genèse $\mathrm{du}$ 《 théâtre indigène ».

La pratique du théâtre, dans les anciennes colonies britanniques, semble jouir d'une plus grande autonomie dans la conception du jeu et dans le statut d'acteur et du dramaturge en raison d'un travail de régulation et de canonisation des mécanismes socioculturels qui assurent aux manifestations spectaculaires un dynamisme particulier peu enclin à la vénération d'Aristote. Très vite, le théâtre est pris en charge par la critique universitaire qui en évalue le langage et la morphologie en réfléchissant sur les procédés 
non conventionnels et les structures scéniques des rites, des réjouissances populaires. Déjà, en 1962, l'université d'Ibadan, au Nigeria, sera la première en Afrique à fonder un département d'art dramatique : School of Theatre Arts qui vient suppléer au projet de promotion de l'art dramatique amorcé par la WNTV (Western Nigerian Television) depuis sa création en 1959. Le petit écran devient le support du théâtre nigérian dans lequel les langues locales comme le yoruba, le ibo ont pris leur revanche sur la langue anglaise, elle-même fortement décolorée par le pidgin véhiculaire ${ }^{36}$. Des dramaturges et comédiens comme Duro Ladipo, Ogunde, Jimoh Buraimoh, Agbo Folarin sont constamment présents sur le petit écran où, à travers leurs prestations, ils tournent en dérision certaines habitudes de pensée et de comportements, de gouvernance, qui infestent la communauté. C'est par ce biais que le futur prix Nobel Wole Soyinka s'est révélé au public. Sa pièce My Father's Burden est passée sur les écrans de la WNTV dans les années 1960.

Depuis lors, les chaînes de télévision se sont multipliées au Nigeria comme The Delta State Television, le NTA (National Television Authorithy), etc. qui font la part belle au théâtre télévisé mettant ainsi constamment les populations au contact des plus grands débats politiques, économiques, culturels de leurs temps. Le théâtre doit alors son dynamisme à l'interaction d'un public cible complice avec lequel il partage ses codes. L'exemple nigérian fait école dans la plupart des pays anglophones où la pratique du théâtre, dès ses débuts, implique une intentionnalité communicative entre le peuple et ses propres représentations, entre la scène - travail de réinvention du réel - et la réalité culturelle et cultuelle où se joue le premier niveau de dédoublement de l'individu entre transcendance et performance. Au Ghana, par exemple, le répertoire des différentes troupes créées au lendemain des indépendances (1957) comme The Abibigroma National Drama Group, National Danse Ensemble, National Symphony Orchestra et Awareness Theatre International s'inspire largement de la tradition des Anansesem (récits de l'Araignée), un art particulier de narration et d'anecdotes au sein duquel trône le personnage d'Ananse (Araignée), métaphores des écarts de comportements par rapport aux normes morales. Outre ces troupes itinérantes qui puisent l'art scénique du théâtre populaire du Concert party, nombreux sont les dramaturges qui prennent comme paradigmes à leurs créations les légendes, les récits et les rituels populaires dans lesquels se retrouve aisément le public. On notera particulièrement le travail de Christiane Ama Ata Aïdo dont les œuvres comme The Dilemma of a Ghost (1956), Anowa (1970) prennent leur racine dans des légendes ashantie. Efua Sutherland, à qui l'on doit la création du Ghana Drama Studio au début des années 1960, initie un théâtre également nourri 
aux sources des contes et des folklores du terroir comme cela se donne à apprécier entre autres dans The Marriage of Anansewa (1975).

Le système colonial semble avoir favorisé dans les milieux anglophones une prise de conscience de soi d'où est né un affrontement permanent entre Soi et Soi comme un Autre, situation d'auto-représentation qui débouche nécessairement sur l'invention des formes esthétiques qui disent la joie, le malaise, le drame, l'angoisse ou les rêves de la société vue de l'intérieur.

Considérer le théâtre anglophone dans sa singularité et dans les perspectives référentielles ainsi définies, c'est marquer que le théâtre en Afrique peut être aussi étudié sur un plan purement horizontal en confrontant les pratiques rituelles, les réjouissances populaires à leur processus de transformation par des personnes qui en font leur métier ; c'est marquer qu'en dehors des apports verticaux entretenus avec l'Europe, le théâtre reste un langage social et un mécanisme de socialité en Afrique même et dont on pourrait étudier les schèmes et les schémas d'évolution. Une nouvelle approche de la pratique de la scène dans ses généralités et ses singularités est nécessaire par-delà le motif de la langue (anglophone, francophone, lusophone) qui a ceci de pernicieux qu'elle privilégie le texte littéraire au détriment du jeu, relègue d'autres formes de théâtre dans le folklore, exile le théâtre dans une sphère où l'art se fait un produit de luxe pour bourgeoisie locale ou encore en fait un art d'exportation.

En effet, la constellation des mécanismes d'invention, de conception de la scène dans les différentes cultures africaines prouve qu'en dehors des multiples formes de manifestations spectaculaires, le concept de théâtre propose un double paradigme : un théâtre du dedans et un théâtre du dehors ; un théâtre intérieur et un théâtre extérieur dont les études pourraient autoriser un regard autre sur le rapport scène-public.

Théâtre du dedans s'entend : manifestation rituelle de transe, de possession dans laquelle le corps de l'acteur démiurgique fait office de lieu scénique ou joue en acteur l'esprit possesseur. Ce théâtre est réservé à un public capable d'identifier, par des codes appropriés, l'acteur invisible dont la performance transite par le corps inconscient du possédé. C'est dire qu'il y a un degré de communication et de connaissance entre le public et l'indétermination jouissive de ses divinités qui ne sont ni substance ni idée mais des représentations de l'esprit. Le spectateur se doit d'être un connaisseur averti pour se représenter, au-delà de l'être en chair et en os qui n'a pas conscience de son jeu, la force incarnée qui agit en lui et dont les langages corporel et verbal sont bien descriptibles. Il ne s'agit pas ici du phénomène anthropologique de la transe 
ou de la possession mais de la représentation que le public en fait devant une manifestation qui participe du rituel et du ludique.

\section{Conclusion}

À côté du théâtre intérieur existe un théâtre extérieur qui résulte d'un faceà-face entre l'acteur et le public, le premier étant maitre de son jeu, le second souscrivant aux conventions d'une esthétique donnée. C'est le traditionnel scène-public proprement dit qui se fonde sur un certain nombre de conventions dont la première est la conscience du jeu unanimement partagée. Il peut être la forme maitrisée du théâtre intérieur à travers une logique de personnage, une dissolution du rite qui dégage l'espace profane du ludique. Comment alors la dramaturgie, surtout populaire, combine-t-elle les deux réalités du théâtre du dedans et du théâtre du dehors comme une maîtrise de la transe, des performances de l'invisible esprit possesseur ? Comment créet-elle un rapport nouveau entre public, acteur-comédien, poète-dramaturge ? Comment se mettent en place les outils critiques qui accompagnent cette métamorphose en art constitué des manifestations spectaculaires et quelle en est la fonctionnalité ? Telles sont certaines des questions sur lesquelles il convient de revenir pour mieux dégager la spécificité des théâtres africains par-delà la littérature dramatique et la lourde ombre d'Aristote.

Devant un théâtre peu tourné vers ses habitudes et son mode de représentation du monde, le public éprouve un sentiment de crise d'identité dès lors que le théâtre vu n'est pas son théâtre vécu. Il ne s'agit pas tant de ce que l'on a désigné au début des années 1970 avec le théâtre d'Hélène Cixous comme « le théâtre du quotidien » que de ménager psychologiquement dans la dramaturgie une place et un rôle extrêmement discret au public. Le texte est un possible du théâtre, sa mise en parole gagne en intensité en rencontrant le langage du public, d'où se comprend l'attitude de révolte et de déception du public d'un théâtre dans lequel il est étranger.

La remarque invite à approcher le théâtre africain comme un art qui tire sa substance de la pluralité des esthétiques et dont les motifs et les langages naissent d'un entrecroisement culturel avec le reste du monde. Le système éducatif et les enseignements du théâtre, surtout dans l'aire francophone, se constituent essentiellement d'une description des champs traditionnels des différents genres : la tragédie, la comédie, le drame, etc. détournant et se détournant par là même de ce qui se fait sur le terrain en matière de création dramatique. D'où la dualité d'un théâtre intérieur - théâtre populaire et téléthéâtre limités à des espaces de fortune mais intensément appréciés du grand 
public - et d'un théâtre extérieur confusément en quête de public et qui, s'il n'est pas condamné aux différents centres culturels et aux rares infrastructures des grandes villes - notamment la capitale -, devient fatalement un théâtre d'exportation. Car à quoi sert le théâtre si le monde qu'il est censé représenter n'est pas ou ne peut pas être présent à la représentation ? C'est ainsi que le « regard étranger » de l'Occident, comme l'est celui d'Aristote même sur le théâtre à Athènes, a longtemps desservi la pratique théâtrale en Afrique, en la réduisant à sa portion congrue. Et pour Florence Dupont :

Si Aristote peut aussi bien objectiver le théâtre et le réduire à un texte, c'est qu'il n'était pas athénien et n'avait donc jamais célébré les Grandes Dionysies; il n'avait jamais chanté dans les chours, jamais été juré, jamais appartenu à ce public athénien qui était initié depuis l'enfance au code musical, en célébrant chaque année ce rituel de la cité d'Athènes, singulier et identitaire ${ }^{37}$.

Il y a nécessité de domestiquer le théâtre en Afrique, ce qui revient sans nul doute à comprendre qu'il est fondamentalement une double représentation : car scène du vécu sur le plan du jeu scénique, il peut aussi s'approprier l'espace du texte et du discours où, recherchant une réévaluation de son image, le critique de théâtre, le dramaturge, l'acteur, engage sa propre scène : celle du sujet du discours.

\section{Notes}

1. Florence Dupont, Aristote ou le vampire du théâtre occidental, Paris, Flammarion, «Libelles », 2007, quatrième de couverture.

2. Johan Huizinga, Homo ludens. Essai sur la fonction sociale du jeu, Paris, Gallimard, 1951, p. 28.

3. Ibid., p. 37.

4. Jean Duvignaud, op. cit., p. 163.

5. Ibid., p. 63-64.

6. Bakary Traoré, Le Théâtre négro-africain et ses fonctions, Paris, Présence Africaine, 1958, p. 23.

7. «La parole proférée au théâtre se présente le plus souvent sous la forme du dialogue dans lequel les répliques des personnages alternent », G. Girard, R. Ouellet, C. Rigault, L'Univers du théâtre, Paris, PUF, $3^{e}$ édition revue et corrigée, 1995 [1978], p. 37.

8. Bernard Agudze-Vioka, Mensonges en or, Lomé, Éditions Haho, 2001, p. 30.

9. Pierre-Aimé Touchard, L'Amateur de théâtre ou la règle du jeu, Paris, Seuil, 1952, p. 12.

10. Ayayi Togoata Apédo-Amah, "L'attitude spectatrice du public africain », Palabres, vol. 2, nos 1 et 2, «Théâtres d'Afrique et des Caraïbes », juin 1998, p. 85-92.

11. Ibid., p. 91.

12. La querelle entre les partisans de la conception du théâtre comme art lié au texte (Aristote, Auguste Conte, Henri Hytier, Lucien Dubech, etc.) et les partisans de la conception 
d'un théâtre limité au spectacle (Edward Gordon Graig, Antonin Artaud, etc.) ne date pas d'aujourd'hui. Et pourtant, c'est à partir de la première conception forte critiquable dans une tradition de l'oralité - par laquelle tout peuple accède à la littérature - que Charles Béart et plus tard tous les exégètes de la dramaturgie africaine vont dater le théâtre africain avec l'expérience de William Ponty. Tout s'est passé comme si William Ponty était le lieu générateur d'une dramaturgie africaine exactement comme si on tentait de faire croire que Thespis, Euripide, Sophocle, etc. avaient apporté l'art dramatique à la Grèce antique. Notre hypothèse est que le corps est l'origine et le lieu premier de la dramaturgie.

13. Henry Brunschwig, Mythes et réalités de l’impérialisme colonial français (1871-1914), Paris, Armand Colin, 1960.

14. François Victor Equilbecq, La Légende Samba Guélâdio Diègui, prince du Fouta, DakarAbidjan, NEA, 1974, p. 146.

15. Voir Apédo-Amah Ayayi Togoata, Peinture sociale et théâtre populaire en Afrique noire, thèse pour le doctorat de troisième cycle présentée à l'Université de la SorbonneNouvelle Paris III, 1980, p. 10 sq.

16. Robert Cornevin, Le Théâtre en Afrique et Madagascar, Paris, Le Livre africain, 1970.

17. Johan Huizigan, L’homo ludens, Paris, Gallimard, 1938, rééd. 1951.

18. Florence Naugrette propose de s'en tenir au concept anglais de «performance " plus adéquat à rendre le caractère événementiel de la chose dramatique que l'équivalent français de représentation qui ne distingue pas l'événement du processus par lequel celui-ci se charge de représenter un spectacle (Florence Naugrette, Le Plaisir du spectateur de théâtre, Rosny-sous-Bois, Bréal, 2002, p. 67 et sq).

19. Roger Caillois, Les Jeux et les hommes, Paris, Gallimard, « Idées », 1958.

20. Ibid., p. 136.

21. Johan Huizinga, op. cit., p. 35.

22. Charles S. Peirce, Écrits sur le signe, rassemblés, traduits et commentés par Gérard Deledalle, Paris, Seuil, «L'ordre philosophique », 1978.

23. Au départ, remarque Anne Übersfeld, l'objet n'est pas un signe, il le devient sur la scène, de par sa fonction à l'intérieur de l'ensemble des signes de la représentation » (Anne Übersfeld, "Pédagogie du fait théâtral ", Théâtre mode d'approche, Bruxelles, Laber, 1987, p. 192.).

24. Tzvetan Todorov, Critique de la critique, Paris, Seuil, 1984, p. 188.

25. Koffi Kwahulé, Pour une critique du théâtre ivoirien contemporain, Paris, L'Harmattan, 1996.

26. Le Theatrum Mundi place l'idée que le monde est un théâtre où se joue la destinée humaine à l'échelle de la transcendance alors que le théâtre du représentant vient du fait que la scène est perçue comme une miniaturisation du réel et l'acteur comme une image de sa société.

27. Partant du principe que l'idée de signification est importante dans l'approche des identités collectives, Jacques Berque plaide pour l'avènement d'une sémantique sociale qui prendrait en charge les signes qui opèrent dans le système des représentations. Il en arrive à ces interrogations : « L'acte social, en vrai, ne consiste-t-il pas justement dans les conversions perpétuelles du fait en signe, de la quantité en qualité, des contenus en forme et la réciprocité ? N'est-il pas cette convertibilité même ? Une identité collec- 
tive ne serait-elle pas l'horizon où s'exerce cette convertibilité ? ", "Qu'est-ce qu'une identité collective ?» in Mélanges offerts à Lévi-Strauss, cité par Henri Michaud dans "L'ethnotype comme système de significations », dans Guy Michaud (dir.), Identités collectives et relations inter-culturelles, Bruxelles, Complexes, 1978, p. 28 (p. 19-34).

28. Guy Michaud, «L'ethnotype comme système de significations », dans id., Identité collectives et relations inter-culturelles, Bruxelles, Complexes, 1978, p. 28.

29. Edmond Marc-Lipiansky, "Groupe et identité ", dans Identités collectives et relations inter-culturelles, ouvrage cité, p. 82 (p. 59-88).

30. Éric Landowsky, Présence de l'autre, Paris, PUF, 1997, p. 16.

31. Ibid., p. 26.

32. Jean-Pierre Miquel, Le Théâtre des acteurs. Ces étranges animaux, Paris, Flammarion, 1996, p. 79.

33. Wolf Lorenz, Carl Spieß, Dietrich Westermann, Wiegräbe, etc.

34. Voir le tableau du déroulement des concours reproduit par Vincent Foutchantsé dans son article "Promouvoir une littérature africaine ", in Présence Africaine, n 69, troisième trimestre 1968, p. 128-133.

35. On pourra lire sur ce point précis de l'analyse, l’étude : Sélom Gbanou, « Entre Aristote et la Bible : Toko Atolia un exemple de tragédie en langue africaine », Palabres, vol. 2, $\mathrm{n}^{\text {os }} 1$ et 2,1998 , p. 63-76.

36. Voir le numéro spécial de Palabres consacré à la mise en scène de la langue du dominateur dans l'espace du dominé : «Pidginisation et créolité en littérature africaine et antillaise ", Palabres, vol. 2, n 3, juillet 1999.

37. Florence Dupont, op. cit., p. 32. 